\title{
2.2 CHEST RADIOGRAPH INTERPRETATION
}

Chest radiograph (or chest $\mathrm{x}$-ray; CXR) uses low-level radiation to form an image of the chest anatomy. It is a noninvasive and readily available radiologic study that is an integral part of the initial evaluation of patients with known or suspected cardiopulmonary pathology. It is also a valuable tool to monitor treatment response or to determine interval change for a variety of cardiopulmonary disorders. The CXR is the most common diagnostic $\mathrm{x}$-ray examination, and more than 20 million CXRs are performed annually in US emergency departments. ${ }^{1,2}$ Hospitalists interpret the results of CXRs, often before radiologists, to diagnose, assess disease severity, and develop treatment plans in hospitalized patients.

\section{KNOWLEDGE}

Hospitalists should be able to:

- Explain the normal anatomy of the thorax with particular attention to spatial relationships.

- Describe the patterns seen on CXR, including those of bone and soft tissue structures, airway, lungs, cardiac structure and silhouette, aorta, and diaphragm.

- Explain the indications, limitations, alternatives, and potential adverse effects of CXR.

- Compare the indications and limitations of a portable CXR study with those of a standard study.

- Explain the indications for ordering CXR with special views or patient position.

- Describe the effects of film exposure, inspiratory effort, and patient position on the CXR image.

- Explain the effects of various abnormal processes on the CXR image.

- Explain the limitations of various CXR findings.

\section{SKILLS}

Hospitalists should be able to:

- Identify normal variants on CXR.

- Identify abnormalities on CXR and, when possible, cor- relate the results with the patient's clinical presentation and findings.

- Synthesize CXR findings with other clinical and diagnostic information to diagnose disease and develop a clinical plan.

- Communicate with patients and families to explain results of CXRs and how the findings influence the care plan.

\section{ATTITUDES}

Hospitalists should be able to:

- Prioritize prompt interpretation of CXRs.

- Recognize the value of comparing the current CXR with historical CXR images, when available.

- Adopt a standardized and consistent approach to interpreting CXR images.

- Consult and work collaboratively with radiologists in interpreting complex CXRs and in ordering further diagnostic studies or procedures on the basis of CXR interpretation.

\section{SYSTEM ORGANIZATION AND IMPROVEMENT}

To improve quality and efficiency within their organizations, hospitalists should:

- Lead, coordinate, and/or participate in multidisciplinary initiatives to optimize resource use.

- Lead, coordinate, and/or participate in efforts directed towards system improvements related to the acquisition and interpretation of CXR for hospitalized patients.

- Lead, coordinate, and/or participate in patient safety programs designed to coordinate care transition and the handoff of pending study results at the time of hospital discharge.

\section{References}

1. McCaig LF, Burt CW. National Hospital Ambulatory Medical Care Survey: 2002 emergency department summary. Adv Data. 2004;340:1-34.

2. National Heart, Lung, and Blood Institute. What Is a Chest X-Ray? Available at: www.nhlbi.nih.gov/health/health-topics/topics/cxray. Accessed May 2015. 